\title{
Steady states for Streater's energy-transport models of self-gravitating particles
}

\author{
Piotr BILER ${ }^{1}$, Jean DOLBEAULT ${ }^{2}$, Maria J. ESTEBAN ${ }^{2}$, \\ Peter A. MARKOWICH ${ }^{3}$, Tadeusz NADZIEJA ${ }^{4}$ \\ ${ }^{1}$ Instytut Matematyczny, Uniwersytet Wrocławski, \\ pl. Grunwaldzki 2/4, 50-384 Wrocław, Poland \\ Piotr.Biler@math.uni.wroc.pl, \\ ${ }^{2}$ Ceremade, Université de Paris-Dauphine, \\ pl. de Lattre de Tassigny, 75775 Paris, France \\ \{dolbeaul, esteban\}@ceremade.dauphine.fr \\ ${ }^{3}$ Institut für Mathematik, Universität Wien, \\ Boltzmanngasse 9, A-1090 Wien, Austria \\ Peter.Markowich@univie.ac.at, \\ ${ }^{4}$ Instytut Matematyki, Politechnika Zielonogórska, \\ ul. Podgórna 50, 65-246 Zielona Góra, Poland \\ T.Nadzieja@im.pz.zgora.pl
}

August 18, 2001

\begin{abstract}
We review Streater's energy-transport models which describe the temporal evolution of the density and temperature of a cloud of gravitating particles, coupled to a mean field Poisson equation. In particular we consider the existence of stationary solutions in a bounded domain with given energy and mass. We discuss the influence of the dimension and geometry of the domain on existence results.
\end{abstract}

Key words and phrases: Streater's models, drift-diffusion systems, energy, existence and uniqueness of stationary solutions, Poisson-Boltzmann-Emden equation.

2000 Mathematics Subject Classification: 35Q, 35J60, 82C 


\section{Introduction}

Recently, R. F. Streater derived systems of partial differential equations that describe the dynamics of Brownian particles in the presence of an external potential as well as the accompanying thermodynamic processes, cf. [39, 40, 41] (and [42] for further extensions), as generalizations of the classical Smoluchowski equation (cf. [38]). The paper [6] gave an extension of Streater's models to the case of self-interacting particles via a Poisson type coupling. Also, we refer to [22], where a review of related energy-transport models in solid state physics is given.

The fundamental property that these models share is that they preserve the mass or charge, the energy (so that they satisfy the first law of thermodynamics), and that they are compatible with the second law of thermodynamics.

The models introduced by Streater extend classical Nernst-Planck-Debye-Hückel drift-diffusion systems for charged particles (cf., e.g., [4]) and those for gravitationally attracting particles (cf. [44, 8]) that do not take into account the evolution of the temperature. Mathematical properties of solutions of the isothermal models are quite well understood now. In particular, finite time blow-up of solutions may occur for the models of attracting particles $([7,3,8])$, while solutions for electrically interacting particles are global in time and tend to steady states ([4]).

Systems governing the evolution of the density $u \geq 0$ (or rather densities $u_{1}, \ldots, u_{N}$ of different species) of a cloud of particles consist of equations of two different types. The first group of equations (formally parabolic) takes into account the Brownian diffusion of particles and their collective motion caused by the gradient of a mean field potential. The second equation (of nonclassical type) represents the balance of heat and involves terms connected with thermal diffusion, convection and heat production. The potential consists of a given external one, and either the electric or the gravitational potential generated by the particles themselves. The study of the case of repulsive electric interaction was started recently in [2], where the steady states and time asymptotics of a solution of the evolution problem have been considered. Here we will deal with the case of gravitationally attracting particles.

We remark that the energy-transport models are valid in a transition regime between a fully kinetic (or hydrodynamic) and an isothermal situations. The Cauchy problem of a parabolic model for which the temperature 
is globally defined and which modelizes the relaxation to the same stationary states as in Streater's models has been studied in [36].

In this paper we will focus on the existence and (non)uniqueness of stationary solutions for fixed mass and energy in the gravitational case (cf. previous results in $[25,30])$. The existence of solutions of the Cauchy problem is only partially known and a proof of a global existence result is open and seems very difficult (cf. comments in [6] and [2]). Here we review known results, present case studies (special geometries) and extend the existing theory.

Before going further, let us point out a few references which are relevant for our purpose (but the list is far from being exhaustive). Our study is centered around the Poisson-Boltzmann-Emden equation (see for instance [1] and references therein) which arises in the context of statistical mechanics of gravitating systems (see [33] for an introduction). Standard results on the Poisson-Boltzmann-Emden equation go back to Emden and Fowler (we refer to $[10,19]$ for classical results). The study of bounded radial solutions in balls uses results on the branches which have been established in [21].

In the two-dimensional case, the Poisson-Boltzmann-Emden equation also appears in the context of Onsagers's approach to turbulence for the Euler equation (see $[32,27,23,11,26]$ and more specifically $[17,11]$ in the microcanonical framework). For star-shaped domains Pohozaev's identity (see [34]) is of great importance, for linear equations this method actually goes back to Rellich: see [35]. It is a crucial tool for the study of branches of smooth solutions as well as for singular solutions (see [28, 18] and references therein).

\section{The equations}

We consider the system of parabolic-elliptic equations for the density $u \geq 0$ of a cloud of gravitationally interacting particles, the temperature $\theta>0$ and the potential $\phi$

$$
\left\{\begin{array}{l}
u_{t}=\nabla \cdot\left[\kappa\left(\nabla u+\frac{u}{\theta}\left(\nabla \phi+\nabla \phi_{0}\right)\right)\right] \\
\begin{array}{c}
u \theta)_{t}=\nabla \cdot(\lambda \nabla \theta)+\nabla \cdot\left[\kappa\left(\theta \nabla u+u \nabla \phi+u \nabla \phi_{0}\right)\right] \\
\quad+\left(\nabla \phi+\nabla \phi_{0}\right) \cdot\left[\kappa\left(\nabla u+\frac{u}{\theta}\left(\nabla \phi+\nabla \phi_{0}\right)\right)\right] \\
\Delta \phi=u .
\end{array}
\end{array}\right.
$$


The above system is considered in a bounded connected domain $\Omega \subset \mathbb{R}^{d}$, and supplemented by the boundary conditions

$$
\begin{cases}\partial_{\nu} u+\frac{u}{\theta}\left(\partial_{\nu} \phi+\partial_{\nu} \phi_{0}\right)=0 & \text { (no mass flux) } \\ \partial_{\nu} \theta=0 & \text { (no heat flux) }\end{cases}
$$

where $\partial_{\nu}$ denotes the normal outgoing derivative on the boundary $\partial \Omega$. For the potential $\phi$ we consider either the Dirichlet boundary condition

$$
\phi=0 \quad \text { on } \partial \Omega
$$

or the "free" condition

$$
\phi=E_{d} * u_{\Omega},
$$

where $u_{\Omega}(x)=u(x)$ for $x \in \Omega, u_{\Omega}(x)=0$ for $x \notin \Omega$, and $E_{d}$ is the fundamental solution of the Laplacian in $\mathbb{R}^{d}$. It is reasonable to assume that the given function $\phi_{0}$ satisfies $e^{-\phi_{0} / T} \in L^{1}(\Omega)$ for some $T>0$, i.e. the external potential $\phi_{0}$ is confining at the temperature $T$.

Note here that we could also consider the case of an unbounded domain (eventually the whole space) for which the confinement is entirely due to the external potential (see [9] for examples in the isothermal case). The boundary conditions (2) and (3)/(4) then have to be replaced by appropriate growth conditions on the external potential, at infinity. Actually, there is a balance between $\phi_{0}$ and the geometric properties of the domain. For the simplicity of the analysis, we shall consider here a bounded domain without external potential, but physically the other situation (unbounded domain and external potential) is certainly more realistic. In some sense the "free" boundary condition corresponds to an external potential which is constant inside the domain and infinite outside, and at least at a qualitative level our results should extend to a less singular external potential. Concerning the confinement, also note that many results for stationary solutions of gravitational systems are similar to stationary states of charged particles (with the same charge) in the presence of an external potential (see [15]).

The case of Dirichlet boundary conditions is somehow academic but allows computations that we hope to be relevant for more realistic boundary conditions, like for instance the "free" boundary condition. What we have in mind here is the description of a localized gravitational structure, which is confined by an external mechanism which is not described in the model.

The analysis of the (physically relevant) free condition (4) is slightly different in some aspects and will be considered elsewhere. 
The coefficients $\kappa, \lambda$ are nonnegative functions of $x, u, \theta, \phi$, which can vanish only at $\theta=0$.

The Cauchy problem for the system (1), (2) and either (3) or (4) consists in finding a solution $\langle u, \theta, \phi\rangle$ with prescribed initial data $u(x, 0)=u_{0}(x)$ and $\theta(x, 0)=\theta_{0}(x)$.

The boundary conditions (2) guarantee that the total mass $M=\int_{\Omega} u d x$ and the total energy

$$
E=\int_{\Omega} u\left(\theta+\phi_{0}+\frac{1}{2} \phi\right) d x
$$

are preserved for classical solutions. We define also the entropy by

$$
W=\int_{\Omega} u \log \left(\frac{u}{\theta}\right) d x .
$$

For sufficiently smooth solutions of (1)-(2) and either (3) or (4), the entropy $W$ is decreasing and the following production-of-entropy formula holds

$$
\frac{d W}{d t}=-\int_{\Omega} \lambda \frac{|\nabla \theta|^{2}}{\theta^{2}} d x-\int_{\Omega} \kappa u\left|\frac{\nabla u}{u}+\frac{1}{\theta}\left(\nabla \phi+\nabla \phi_{0}\right)\right|^{2} d x .
$$

The entropy production relation (5) implies that for steady states $\langle u, \theta, \phi\rangle$ $\theta=$ const, and the flux $\nabla u+\frac{u}{\theta} \nabla\left(\phi+\phi_{0}\right)$ vanishes a.e. in $\Omega$, so that $\nabla\left(u e^{\left(\phi+\phi_{0}\right) / \theta}\right)=0$. Thus $u$ has the Boltzmann-distributed form

$$
u=C e^{-\left(\phi+\phi_{0}\right) / \theta},
$$

where $C=M\left(\int_{\Omega} e^{-\left(\phi+\phi_{0}\right) / \theta} d x\right)^{-1}$, since $\int_{\Omega} u d x=M$.

\section{The Poisson-Boltzmann-Emden equation}

The case of gravitational interaction is much more complicated than the electric case studied in [2], where the third equation of the system (1) is replaced by the repulsive mean field coupling $-\Delta \phi=u$. The reason is that, contrary to the case of electric interaction, the potential $\phi$ could be (very) negative in the attractive case, so that neither the energy nor the 
entropy relations give reasonable a priori bounds on $\langle u, \theta, \phi\rangle$. From a more mathematical viewpoint, the energy is no more a convex function of $u$ because of the sign of the potential energy term. Even if the external potential $\phi_{0}$ is absent, the structure of the set of steady states (existence-uniqueness vs. nonexistence-multiplicity), determined by the (scaled) Poisson-BoltzmannEmden equation (7) below, is complicated and depends in a very sensitive manner on geometric properties of the domain, cf. [11], [14], and [13] for the case of Riemannian manifolds. For the case of an external (singular) potential, see, e.g., [8]. Typically, variational methods can be used in the twodimensional case (see Section 4), while they are of lesser use for $d>2$ (the case studied in Section 5), cf. [31] and remarks in [44]. For a refined analysis of the problem in three dimensions close to a planar one via variational methods, we refer the reader to the recent paper [26].

Now we study stationary solutions of $(1)$, with $\phi_{0} \equiv 0$, in a bounded domain $\Omega \subset \mathbb{R}^{d}$, i.e.,

$$
u \equiv \Delta \phi=M \frac{e^{-\phi / \theta}}{\int_{\Omega} e^{-\phi / \theta} d x} \quad \text { in } \Omega,
$$

(see (6)), supplemented by the Dirichlet boundary condition (3).

Although the equation (7) as steady state equation for the gravitational Streater's model has a physical meaning only on 1, 2 or 3-dimensional domains, we shall in the sequel consider its scaled version (9) subject to (8)-(10) on domains of arbitrary dimension $d$. The main reason for this is that the most important mathematical properties do not change for $d$ larger or equal 3. Also we believe that more mathematical structure is revealed by this generality.

Scaling the potential solving (7) with (3) as $\phi=\theta \psi$, the energy becomes $E=M \theta+\frac{1}{2} \theta^{2} \int_{\Omega} \psi \Delta \psi d x=M \theta-\frac{1}{2} \theta^{2} \int_{\Omega}|\nabla \psi|^{2} d x$. The problem of finding a solution of (7) with given energy $E$ and mass $M>0$ is equivalent to looking for a solution of the equation

$$
\left(\frac{E}{M^{2}}\right) m^{2}=m-\frac{1}{2} \int_{\Omega}|\nabla \psi|^{2} d x \equiv \mathcal{E}(m, \psi) .
$$

Here $m=M / \theta$ and $\psi$ solves the Poisson-Boltzmann-Emden equation

$$
\Delta \psi=m \frac{e^{-\psi}}{\int_{\Omega} e^{-\psi} d x} \quad \text { in } \Omega,
$$


subject to the condition

$$
\psi=0 \quad \text { on } \partial \Omega
$$

in the range of admissible $m>0$, and satisfies $E / M^{2}=\mathcal{E}(m, \psi) / m^{2}$ for some $\psi$ solution of (9)-(10). In cases in which this problem does not have a unique solution, it is useful to define

$$
\mathcal{F}(m)=\inf _{\psi \text { solutions of }(9)-(10)} \mathcal{E}(m, \psi),
$$

where (9) is the Poisson-Boltzmann-Emden equation, with parameter $m$.

Some of the results on the problem (8)-(10) in Section 4 (in the twodimensional case) are consequences of subtle properties of solutions of the Poisson-Boltzmann-Emden equation (9)-(10) proved in [11].

As it is well known (compare also examples below), (9)-(10) has nontrivial bounded solutions either for $m \in\left(0, m_{\Omega}\right)$ or $m \in\left(0, m_{\Omega}\right]$ with some $0<$ $m_{\Omega} \leq \infty\left(m_{\Omega}\right.$ may be defined as the supremum of the interval $I$ containing 0 such that for any $m \in I$, the Poisson-Boltzmann-Emden Dirichlet problem (9), (10) has at least one bounded solution). In the first case, typically, the density becomes unbounded in $L^{\infty}(\Omega)$ as $m \nearrow m_{\Omega}$. Moreover, in the case of star-shaped domains $\Omega \subset \mathbb{R}^{d}, d \geq 2$, one has $m_{\Omega}<\infty$, while, e.g., for annuli $\Omega \subset \mathbb{R}^{d}, m_{\Omega}=\infty$.

The proofs of nonexistence of solutions of (9)-(10) in star-shaped domains for $m \gg 1$ employ either the Pohozaev identity (in strictly star-shaped domains) or the moment method for the evolution (isothermal) problem. We refer the reader to [11] in the case of the Dirichlet condition and dimension $d=2$. For dimensions $d \geq 3$ see e.g., [16], [7], p. 322 and also [3], Th. 2, and to [7], Th. 2 (v), [3], Th. 1, and [29] in the case of the free condition (4) for $\phi$. We recall an argument based on the Pohozaev method in the proofs of Remark 5.1 and Theorem 5.7. In the two-dimensional case this nonexistence result has been extended to an arbitrary simply connected domain, see [24].

\section{Planar domains}

We emphasize that the results of this section are strongly based on [11], [43]. 
Let us start with an explicit example of the analysis of the problem (8)-(10).

Example 1. If $\Omega$ is the unit disc $B(0,1)=\left\{x \in \mathbb{R}^{2}:|x|<1\right\}$, we have for a fixed $m \in[0,8 \pi)$ a unique radially symmetric solution $\psi_{m}$ of (9)-(10) (see [43]). Moreover, by [20], there is no other bounded solution of this problem. The function $\psi_{m}$ satisfies $\frac{\partial}{\partial r} \psi_{m}=4 r\left(r^{2}+8 \pi / m-1\right)^{-1}$ with $r=|x|$. The direct integration of the equation yields:

$$
\int_{\Omega}\left|\nabla \psi_{m}\right|^{2} d x=2 \pi \int_{0}^{1} r\left(\frac{\partial}{\partial r} \psi_{m}\right)^{2} d r=32 \pi \int_{0}^{1} r^{3}\left(r^{2}+\frac{8 \pi}{m}-1\right)^{-2} d r
$$

which leads to $\lim _{m}>8 \pi \int_{\Omega}\left|\nabla \psi_{m}\right|^{2} d x=\infty$, i.e. $\lim _{m>8 \pi} \mathcal{E}\left(m, \psi_{m}\right)=-\infty$. Since $\inf _{m \in(0,8 \pi)} \mathcal{E}\left(m, \psi_{m}\right)=-\infty$ and $\mathcal{E}\left(m, \psi_{m}\right) \sim m-C m^{2}$ as $m \rightarrow 0^{+}$

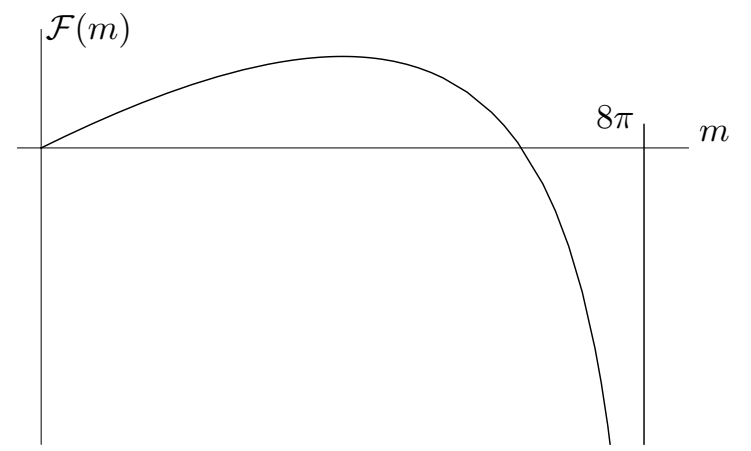

The function $m \mapsto \mathcal{F}(m)=2 m+8 \pi \log \left(1-\frac{m}{8 \pi}\right)$.

for some $C>0$, we have from the analysis of the graph of $\mathcal{E}$ and by the connectedness of the set $\left\{\psi_{m}, m \in[0,8 \pi)\right\}$ (see [43]; see also Remark (5.9)), the following

Proposition 4.1 For each $E \in \mathbb{R}$ and each $M>0$, there exists a solution of (8)-(10) in the unit disc in the plane.

In the two-dimensional case solutions of (9)-(10) can be obtained as the absolute maxima of certain functionals, e.g., $I[\psi]=\log \left(\int_{\Omega} e^{-\psi} d x\right)+$ $\frac{1}{2} \int_{\Omega} \psi \Delta \psi d x$ or $J[\psi]=-\int_{\Omega}(\Delta \psi) \log (\Delta \psi) d x-\frac{1}{2} \int_{\Omega} \psi \Delta \psi d x$ considered in the class of potentials $\psi$ corresponding to the densities $\Delta \psi \geq 0, \int_{\Omega} \Delta \psi d x=m$ and satisfying boundary conditions (10), see [11]. However, there exist steady states that cannot be obtained in this (direct variational) way (cf. [11], 


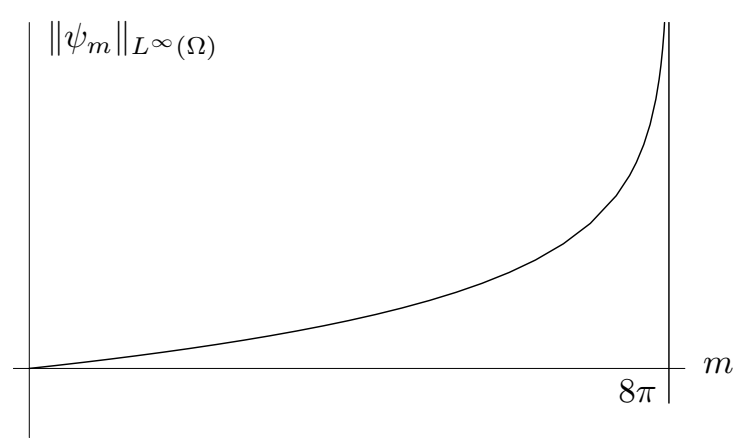

The bifurcation diagram in $(0, \infty) \times L^{\infty}(\Omega)$ when $\Omega$ is the unit disc in $\mathbb{R}^{2}$.

and [14] in non-simply connected domains). The applicability of the direct method of the calculus of variations to the functionals $I$ or $J$ is based on the Moser-Trudinger inequality

$$
\int_{\Omega} \exp (|\psi|) d x \leq C \exp \left(|\Omega|^{-1}\left|\int_{\Omega} \psi d x\right|+|\nabla \psi|_{2}^{2} /(8 \beta)\right),
$$

see [12] and [7], (15.1)-(15.2). The range of the parameter $m$ for which this inequality is useful depends on which boundary condition (3) or (4) is used in domains $\Omega$ with either smooth $\left(C^{2}\right)$ or piecewise smooth boundary $\partial \Omega$, and $\beta=\gamma$ (the minimal interior angle at the vertices of $\partial \Omega$ for (4)), $\beta=2 \gamma$ for (3), while $0 \leq m<4 \beta$ in all these cases. The functionals $I$ and $J$ are also important in the study of global-in-time existence of solutions of the isothermal evolution problem in $[3,4,8]$.

However, these variational principles do not give all the solutions of the Poisson-Boltzmann-Emden problem (9)-(10), e.g. if $m>8 \pi$. The solvability of the homogeneous Dirichlet problem for (7) (and thus (9)-(10)), called also the Mean Field Problem in [11], can be studied in two-dimensional smooth domains using the more general Microcanonical Variational Principle as was done in the second part of [11]. Namely, the maximizer of the entropy functional $S(\varrho)=-\int_{\Omega} \varrho \log \varrho d x$ on the set of densities of nonnegative measures $\varrho \geq 0, \int_{\Omega} \varrho d x=1$, under the constraint of the fixed potential energy $E_{p}=\iint_{\Omega \times \Omega} G(x, y) \varrho(x) \varrho(y) d x d y$ satisfies (7) for $M=1$ and some $\theta$ depending on $E_{p}$. Here $G$ is the Green function for $-\Delta$ on the domain $\Omega$. Note that, corresponding to $m\left(=\frac{1}{\theta}\right)>8 \pi$, there may exist solutions of $(9)-(10)$ satisfying the Microcanonical Variational Principle which are neither maximizers of $I$ nor of $J$, and these solutions are not unique. The nonequivalence 
of the above mentioned variational principles may occur in the, so-called, planar domains of the second kind introduced in [11, Part II, Sec. 6]. Below there is an example of such a domain (its boundary is only piecewise smooth, but it does not matter). A smooth domain of the first kind is, by definition, one with $\inf \{\mathcal{E}(m, \psi): m \in(0,8 \pi), \psi$ solution of $(9)-(10)\}=-\infty$. In domains of the first kind which are simply connected (9)-(10) has a unique bounded solution for each $m \in[0,8 \pi)$, and no bounded solution for $m \geq 8 \pi$. Discs and simply connected domains close to a disc are examples of such domains.

Example 2. (cf. [11], p. 523) If $\Omega \subset \mathbb{R}^{2}$ is a "long" rectangle, i.e. $\Omega=$ $(0, a) \times(0, b)$ with $b \gg a>0$, then $m_{\Omega}<\infty$ but there exists a solution maximizing the functional $I[\psi]$ for $m=8 \pi$, unlike in the case of a disc in the plane. This is a consequence of the relation $\sup _{m \in(0,8 \pi)} I[\psi]<\infty$ which permits us to prove that a maximizing sequence for $I$ with $m \nearrow 8 \pi$ converges to a maximizer of $I$ for $m=8 \pi$. Moreover, there is nonuniqueness of solutions of (9)-(10) : either there are at least two distinct solutions of (9)-(10) for $m=8 \pi$ or there exists a sequence $m_{n} \searrow 8 \pi$ as $n \rightarrow \infty$ such that there are at least two distinct bounded solutions of (9)-(10) for every $m=m_{n}$ (see Theorem 7.1 in [11, Part II]). Here, $\inf _{m \in(0,8 \pi]} \mathcal{F}(m)>-\infty$ is satisfied, while $\inf _{m \in\left(0, m_{\Omega}\right)} \mathcal{F}(m)=-\infty$ still holds. In this case, one can easily see that solutions of (8)-(10) exist for arbitrary $E / M^{2} \in \mathbb{R}$ as was the case for the disc.

Theorem 4.2 If $\Omega$ is a bounded strictly star-shaped domain in $\mathbb{R}^{2}$, with smooth boundary, then for all $M>0$ and $E \in \mathbb{R}$ there exists a solution of (8)-(10).

Proof. The problem (9)-(10) has a solution for $m \in\left[0, m_{\Omega}\right)$, for some $8 \pi \leq$ $m_{\Omega}<\infty$, and has no solution for $m>m_{\Omega}$. Moreover, for each $m \in[0,8 \pi)$ there is a unique bounded solution $\psi_{m}$ and the set $\left\{\psi_{m} \in L^{\infty}(\Omega): 0 \leq\right.$ $m<8 \pi\}$ is connected (see [43]).

It follows from the bound $\|\psi\|_{L^{\infty}(\Omega)} \leq m C(\Omega, m)$ with $C(\Omega, m)$ uniformly bounded as $m \searrow 0$ (cf. [8, p. 187]; see also Lemma 5.3 and Corollary 5.4), that $\mathcal{E}\left(m, \psi_{m}\right) \sim m$ for small $m$.

If $\Omega$ is of the first kind, then $\lim _{m / 8 \pi} \mathcal{E}\left(m, \psi_{m}\right)=-\infty$ and therefore, the problem (8)-(10) has a solution for all $E \in \mathbb{R}$ and $M>0$. 
For domains of the second kind, $\lim _{m / 8 \pi} \mathcal{E}\left(m, \psi_{m}\right)>-\infty$ (cf. [11, p. 251]), but there exists a branch of solutions $\psi_{m}, 0 \leq m<m_{\Omega}$, such that $\inf _{0<m<m_{\Omega}} \mathcal{E}\left(m, \psi_{m}\right)=-\infty$ (see [43] and [11]). This implies the existence of solutions of (8)-(10) in that case (and they may be multiple for $m \geq 8 \pi$, see Theorem 7.1 in $[11$, Part II]).

As we already remarked, problem (9)-(10) in star-shaped domains does not have solutions for large $m$ (see a simple proof of this fact in the next section). However, in certain domains, e.g. the annuli, solutions exist for all $m>0$, as can be seen in [11]. In those cases there exists $\ell_{0} \in \mathbb{R}$ such that there is no steady state of Streater's system (1)-(3) if $E / M^{2}<\ell_{0}$ (see Proposition 5.10). Such results are typical in higher dimensional situations as well as on 1-dimensional intervals.

\section{Higher dimensional problem}

The goal of this section is to analyze the existence of solutions of (8)-(10) in higher dimensions $d \geq 3$. However, several results of this section are also valid in lower dimensions. Our strategy here is to prove that

(i) Near $m=0^{+}, \mathcal{F}(m) \sim m$.

(ii) There exists a constant $\ell_{0} \in \mathbb{R}$ such that $\mathcal{F}(m) \geq \ell_{0} m$.

\subsection{Preliminary results - star-shaped domains}

Let us start with some standard facts and preliminary results.

Note first that (9) is autonomous, so that we may assume without loss of generality that $\Omega$ is star-shaped with respect to the origin as soon as it is star-shaped with respect to some point.

Using $x \cdot \nabla \psi$ as a test function in (9), and integrations by parts, we easily recover Pohozaev's identity

$$
\frac{1}{2} \int_{\partial \Omega}(x \cdot \nu)\left|\partial_{\nu} \psi\right|^{2} d \sigma+\frac{d-2}{2} \int_{\Omega}|\nabla \psi|^{2} d x=d m\left(1-\frac{|\Omega|}{\int_{\Omega} e^{-\psi} d x}\right),
$$

where $\sigma$ is the measure on $\partial \Omega$ induced by the Lebesgue measure. If $\Omega$ is a star-shaped bounded domain (with respect to the origin), then the first term of the left hand side is nonnegative. 
Remark 5.1 Recall a standard fact that if $\Omega$ is a strictly star-shaped bounded domain in $\mathbb{R}^{d}, d \geq 2$, the range of $m>0$ such that (8)-(10) has a bounded solution is bounded: $m_{\Omega}<\infty$.

The argument goes as follows. We assume that $\Omega$ is strictly star-shaped with respect to the origin. Then $\beta=\min _{x \in \partial \Omega} x \cdot \nu(x)>0$. We integrate (9) over $\Omega$ and use the Gauss theorem:

$$
\int_{\partial \Omega} \partial_{\nu} \psi d \sigma=\int_{\Omega} \Delta \psi d x=m
$$

which combined with Hölder's inequality gives

$$
m^{2}=\left(\int_{\partial \Omega} \partial_{\nu} \psi d \sigma\right)^{2} \leq \int_{\partial \Omega}\left|\partial_{\nu} \psi\right|^{2} d \sigma|\partial \Omega|
$$

and thus, according to (12),

$$
d m-\frac{\beta}{2} \frac{m^{2}}{|\partial \Omega|} \geq 0 .
$$

Note that this argument is sharp for a ball in dimension $d=2$ (see $[11,16,24]$ and references therein) and gives for unit balls in $\mathbb{R}^{d}, d \geq 2$, the condition

$$
m \leq m_{\Omega} \leq 2 d \sigma_{d}
$$

where $\sigma_{d}=\left|S^{d-1}\right|$ is the surface of the unit sphere, with a strict inequality if $d>2$ (for the last assertion see, for instance, [7], Sec. 2).

Lemma 5.2 Assume that $\Omega$ is a bounded star-shaped domain in $\mathbb{R}^{d}$, with $d \geq 3$. Then there exists a constant $\alpha_{d}$, such that $\mathcal{F}(m) \geq \alpha_{d} m$. Moreover, if $d \geq 15$, then $\alpha_{d}>0$.

Proof. Consider the equation

$$
\Delta \psi=m \frac{e^{-\psi}}{\int_{\Omega} e^{-\psi} d x} \equiv \varrho .
$$

Taking the logarithm of the right hand side, we have

$$
-\psi=\log \varrho-\log \left(\frac{m}{\int_{\Omega} e^{-\psi} d x}\right) .
$$


Thus

$$
\int_{\Omega}|\nabla \psi|^{2} d x=\int_{\Omega} \varrho \log \varrho d x-m \log \left(\frac{m}{\int_{\Omega} e^{-\psi} d x}\right) .
$$

On one hand, Jensen's inequality gives

$$
\int_{\Omega} \varrho \log \varrho d x \geq m \log \left(\frac{m}{|\Omega|}\right)
$$

and on the other hand, from Pohozaev's identity (12), we know that

$$
\frac{1}{2} \int_{\Omega}|\nabla \psi|^{2} d x \leq \frac{d m}{d-2}\left(1-\frac{|\Omega|}{\int_{\Omega} e^{-\psi} d x}\right) .
$$

A combination of (13), (14) and (15) implies that $X=|\Omega|^{-1} \int_{\Omega} e^{-\psi} d x$ satisfies the inequality

$$
\log X \leq \frac{2 d}{d-2}\left(1-\frac{1}{X}\right)
$$

or, in other words, that

$$
X \in\left[1, X^{*}(d)\right] \quad \text { with } \quad \log X^{*}(d)=\frac{2 d}{d-2}\left(1-\frac{1}{X^{*}(d)}\right)>0 .
$$

It is straightforward that $d \mapsto X^{*}(d)$ is decreasing on $(0, \infty)$. To conclude, we simply use again (15) and write

$$
\begin{gathered}
\mathcal{E}(m, \psi)=m-\frac{1}{2} \int_{\Omega}|\nabla \psi|^{2} d x \geq m-\frac{d m}{d-2}\left(1-\frac{|\Omega|}{\int_{\Omega} e^{-\psi} d x}\right), \\
\mathcal{E}(m, \psi) \geq \frac{m}{d-2}\left(\frac{d}{X^{*}(d)}-2\right) \equiv \alpha_{d} m .
\end{gathered}
$$

With this notation, $\alpha_{d}>0$ if and only if $X^{*}(d)<d / 2$, which according to the definition of $X^{*}(d)$ means $d>2 e^{2} \in(14,15)$.

In particular, the results in Lemma 5.2 exclude the existence of solutions of (7), (3) with negative energies (cf. Th. 5.7) for $d \geq 15$.

If $d=2$, it is possible to prove a uniqueness result for small $m>0$, (see [11] or [8] p. 187). For the completeness of this article, we state the result here, with a short proof. 
Lemma 5.3 Assume that $\Omega$ is a bounded domain in $\mathbb{R}^{2}$. Then there exists an $m^{*} \in\left(0, m_{\Omega}\right]$ such that for any $m \in\left(0, m^{*}\right)$, equation (9) has a unique bounded solution $\psi$, which moreover satisfies

$$
\|\psi\|_{L^{\infty}(\Omega)} \leq m C(\Omega, m)
$$

with $C$ locally bounded in $m \in\left[0, m^{*}\right.$ ) (but possibly $\lim _{m \nearrow m^{*}} C(\Omega, m)=\infty$ ).

Proof. Let $G$ be the Green function of the negative Laplacian in $\Omega$ corresponding to the homogeneous Dirichlet boundary conditions and consider $s$, $s^{\prime} \in(1, \infty)$ such that $1 / s+1 / s^{\prime}=1$. With the notations $\varrho=m \mu^{-1} e^{-\psi}$, $\mu=\int_{\Omega} e^{-\psi} d x$, if $\psi$ is a solution of (9)-(10), we have

$$
-s \psi(x) \leq s m \int_{\Omega}|G(x, y)| \frac{\varrho(y) d y}{m} .
$$

Using Jensen's inequality and integrating with respect to $x$, we obtain

$$
\left\|e^{-\psi}\right\|_{L^{s}(\Omega)}^{s}=\int_{\Omega} e^{-s \psi(x)} d x \leq \int_{\Omega} \frac{\varrho(y) d y}{m} \int_{\Omega} e^{s m|G(x, y)|} d y d x .
$$

Since $\int_{\Omega} \frac{\varrho(y) d y}{m}=1$ and $|G(x, y)| \leq C(|\log | x-y||+1)$ for some constant $C>0,\left\|e^{-\psi}\right\|_{L^{s}(\Omega)}$ is bounded uniformly with respect to $m$ sufficiently small. Next, using Hölder's inequality, it is easy to check that

$$
|\Omega| \leq \mu^{s /(s+1)}\left\|e^{-\psi}\right\|_{L^{s}(\Omega)}^{s /(s+1)},
$$

and hence $0<\mu^{-1} \leq|\Omega|^{-1-1 / s}\left\|e^{-\psi}\right\|_{L^{s}(\Omega)}$. Thus we have

$$
\left\|m \mu^{-1} e^{-\psi}\right\|_{L^{s}(\Omega)} \leq m|\Omega|^{-1-1 / s}\left\|e^{-\psi}\right\|_{L^{s}(\Omega)}^{2} \leq C m
$$

for some constant $C>0$ independent of $m$, which implies

$$
\|\psi\|_{L^{\infty}(\Omega)}=\left\|m \mu^{-1} \int_{\Omega} G(x, y) e^{-\psi(y)} d y\right\|_{L^{\infty}(\Omega)} \leq C m\left(\sup _{x \in \Omega} \int_{\Omega}|G(x, y)|^{s^{\prime}} d y\right)^{1 / s^{\prime}} .
$$

It is easy to check that $\sup _{x \in \Omega} \int_{\Omega}|G(x, y)|^{s^{\prime}} d y$ is finite for any $s^{\prime} \in(1, \infty)$ which, combined with a contraction mapping argument, completes the proof.

In higher dimensions it is possible to replace the inequality (16) by a direct estimate of $\left\|e^{-\psi}\right\|_{L^{s}(\Omega)}$ if one is able to show at least the existence of a uniform 
bound for the density $\varrho$ in an appropriate Morrey space containing $L^{d / 2}(\Omega)$ (see Th. 1 (ii) in [7]). Uniqueness then follows.

Apart from uniqueness questions, for $d=2$, Lemma 5.3 is sufficient to estimate $\mathcal{E}\left(m, \psi_{m}\right)$ for $m$ small.

Corollary 5.4 With the same notations as in Lemma 5.3,

$$
\mathcal{E}\left(m, \psi_{m}\right) \geq m-\frac{1}{2} C(\Omega, m) m^{2} \quad \text { for each } m \in\left[0, m^{*}\right) .
$$

Proof. Under the boundary condition (10),

$$
\int_{\Omega}|\nabla \psi|^{2} d x=-\int_{\Omega} \varrho \psi d x \leq C(\Omega, m) m^{2} .
$$

Going back to the case $d \geq 3$, we will state a result which is valid for bounded solutions only. Note that for such a solution $\psi, e^{-\psi} \in L^{\infty}(\Omega)$, and by a standard iteration method, one gets $\psi \in C^{\infty}(\Omega)$. The regularity up to the boundary depends on the regularity of $\partial \Omega$, cf. [28].

Consider now the solutions $\langle\lambda, \psi\rangle \in[0, \infty) \times L^{\infty}(\Omega)$ to

$$
\left\{\begin{array}{l}
\Delta \psi=\lambda e^{-\psi} \quad \text { in } \Omega, \\
\psi=0 \quad \text { on } \partial \Omega
\end{array}\right.
$$

with $0<\lambda=\frac{m}{\int_{\Omega} e^{-\psi} d x}$. Note that $\lambda \leq \frac{m}{|\Omega|}$, since $\psi<0$ in $\Omega$ by the Maximum Principle. There exists a constant $C>0$, which only depends on the dimension $d \geq 3$ such that, for any bounded solution $\psi$ of (18),

$$
\int_{\Omega}|\nabla \psi|^{2} d x \leq C \lambda
$$

as a consequence of the computations of Lemma 5.2 (also see [28]). Let us state the the following result, due to X. Cabre and P. Majer and cited in [28] (also see [37]).

Theorem 5.5 If $\Omega$ is star-shaped and if $d \geq 3$, there exists $\lambda_{0}>0$ such that for any $\lambda \in\left(0, \lambda_{0}\right)$, there exists a unique bounded solution of (18). 
Corollary 5.6 If $\Omega$ is star-shaped and if $d \geq 3$, there exist a continuous family of bounded solutions $\psi_{m}$. Moreover, there exists two positive constants $C, m^{*}$ such that if $m \in\left(0, m^{*}\right)$ then, for any solution $\psi_{m}, m \geq \mathcal{E}\left(m, \psi_{m}\right) \geq$ $m-C m^{2}$.

Proof. The function $\psi_{m}$ exists by results of [7]. Moreover, applying Theorem 5.5 with $\lambda=\frac{m}{\int_{\Omega} e^{-\psi_{m}} d x} \leq \frac{m}{|\Omega|} \rightarrow 0$ as $m \rightarrow 0$, any bounded solution $\psi_{m}$ is uniformly small in $L^{\infty}(\Omega)$ with respect to $m>0$, small:

$$
1-\frac{|\Omega|}{\int_{\Omega} e^{-\psi_{m}} d x}=O(m) \quad \text { as } m \rightarrow 0^{+},
$$

which proves that $\int_{\Omega}\left|\nabla \psi_{m}\right|^{2} d x=O\left(m^{2}\right)$ as $m \rightarrow 0$.

Theorem 5.7 If $\Omega$ is a bounded star-shaped domain in $\mathbb{R}^{d}, d \geq 3$, with a smooth boundary, then there exists a constant $\ell_{1} \in \mathbb{R}$ such that for any $E / M^{2}>\ell_{1}$, there is a nontrivial bounded solution of the problem (8)-(10), and there exists a constant $\ell_{0}$ such that if $E / M^{2}<\ell_{0}$, then (8)-(10) has no nontrivial bounded solution. Moreover, if $d \geq 15$, there exists a positive constant $\tilde{\ell}_{0}$ such that if $E / M^{2}<\tilde{\ell}_{0}$, then (8)-(10) has no solution in $H_{0}^{1}(\Omega)$.

Note that $\ell_{0} \leq \ell_{1}$, with equality if, for instance, there is a single curve of bounded solutions of (8)-(10).

Proof. By Corollary 5.6 the existence of $\ell_{1}$ follows. On the other hand, Lemma 5.2 and Corollary 5.6 imply the statement concerning $\ell_{0}$.

The case $d \geq 15$ follows from the estimate of Lemma 5.2.

\section{$5.2 \quad$ Examples}

\subsubsection{Balls}

If $\Omega$ is the unit ball $B(0,1) \subset \mathbb{R}^{d}, d>2$, the problem (9)-(10) is no longer integrable as it was the case for $d=2$, but it can be reduced to the study of a dynamical system in the plane (introduced by I. M. Gelfand in [19]), cf. [5] and [8]. Moreover, the set of the solutions can be explicitly parametrized (see Remark 5.9) and forms a single connected branch. Using this idea, one checks that $\inf _{m \in\left(0, m_{\Omega}\right)} \mathcal{F}(m)>-\infty$. This means that (8) does not have solutions for $E / M^{2}$ below some real constant, $\ell_{0}$ (and $\ell_{0}=\ell_{1}$ with the notations of Theorem 5.7). Also remark that if $3 \leq d \leq 9$, the solutions of (9)-(10) for given $m$ are not, in general, unique, see e.g. [5, Sec. 2]. 
Proposition 5.8 There exists $\ell_{0} \in \mathbb{R}$ such that for $E / M^{2}>\ell_{0}$ there is a bounded negative solution of (8)-(10) in the unit ball of $\mathbb{R}^{d}, d \geq 3$, and there are no nontrivial bounded negative solutions if $E / M^{2}<\ell_{0}$.

Proof. According to [20], negative solutions of (8)-(10) in a ball are radially symmetric. For such solutions, we introduce an auxiliary function $Q(r)=$ $\int_{B_{r}} \Delta \psi d x$ (the integrated density). A simple computation shows that $Q$ satisfies the system

$$
\begin{array}{r}
Q_{r r}-(d-1) r^{-1} Q_{r}+\sigma_{d}^{-1} r^{1-d} Q Q_{r}=0, \\
Q(0)=0, \quad Q(1)=m,
\end{array}
$$

and $\frac{d}{d r} \psi(r)=\sigma_{d}^{-1} r^{1-d} Q(r)$, cf. [5, Sec. 2]. Note that this is a boundary value problem for a nonlinear second order differential equation, i.e. it is no longer a nonlocal problem as (9) was. Changing the variables $s=\log r$, $v(s)=\sigma_{d}^{-1} r^{3-d} Q_{r}(r), w(s)=\sigma_{d}^{-1} r^{2-d} Q(r)$, we obtain

$$
v^{\prime}=(2-w) v, \quad w^{\prime}=(2-d) w+v,
$$

where' denotes $\frac{d}{d s}$, together with the boundary conditions $w(-\infty)=0$ and $w(0)=\sigma_{d}^{-1} \mathrm{~m}$. Observe that there is a unique trajectory (a separatrix) such that $w(s) \geq 0$ for $s \rightarrow-\infty$, and which satisfies $w(-\infty)=0$. Since $\lim _{s \rightarrow \infty}\langle w(s), v(s)\rangle=\langle 2,2(d-2)\rangle$, this trajectory is bounded (in fact, $\left.\sup _{s \in \mathbb{R}} w(s)<2 d \sigma_{d}\right)$. A shift of the argument $s$ gives the proper normalization of the value of $w(0)$.

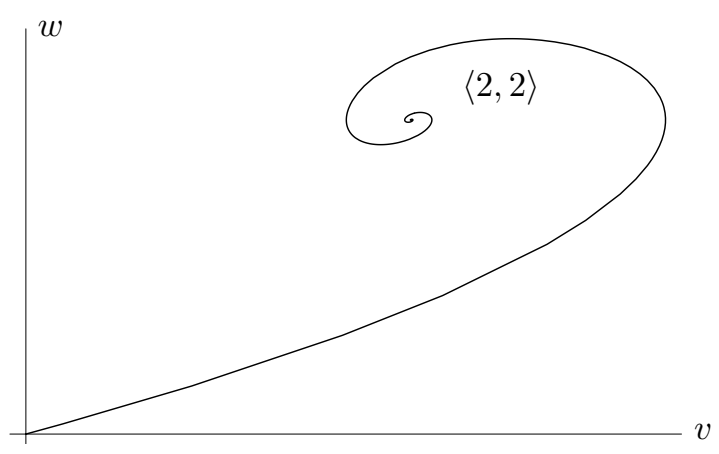

The trajectory $s \mapsto\langle v(s), w(s)\rangle$ with limits $\langle 0,0\rangle$ as $s \rightarrow-\infty$ and $\langle 2(d-2), 2\rangle=$ $\langle 2,2\rangle$ as $s \rightarrow \infty$ in the case $d=3$. 
To prove that for $d \geq 3$ the relation $\inf _{m \in\left(0, m_{\Omega}\right)} \mathcal{F}(m)>-\infty$ holds, we compute

$$
\int_{\Omega}|\nabla \psi|^{2} d x=\sigma_{d}^{-1} \int_{0}^{1} Q^{2}(r) r^{1-d} d r=\int_{-\infty}^{0} w(s) Q(s) d s=\sigma_{d} \int_{-\infty}^{0} w^{2}(s) e^{s(d-2)} d s
$$

which is uniformly bounded for all $m \leq m_{\Omega}<2 d \sigma_{d}$ (by Remark 5.1). We see also that for $d=2$ the above integral is not bounded which was explicitly checked in Example 1.

Remark 5.9 In the case of a ball of $\mathbb{R}^{d}(d>1)$, the shift in $s$ of the trajectory $s \mapsto\langle w(s), v(s)\rangle$ also parametrizes the set of bounded solutions which therefore belong to a simply connected branch. This can be seen more easily in the original variables: for any $a>0$, consider the solution $\varphi_{a}=\varphi$ of

$$
\left\{\begin{array}{l}
\varphi^{\prime \prime}+\frac{d-1}{r} \varphi^{\prime}=e^{-\varphi}, \quad r>0 \\
\varphi(0)=-a, \quad \varphi^{\prime}(0)=0
\end{array}\right.
$$

For any $a>0$, define $m(a)=\sigma_{d} \int_{0}^{1} r^{d-1} e^{-\varphi_{a}(r)} d r$ and $\psi_{a}(r)=\varphi_{a}(r)-$ $\varphi_{a}(1)$. Then $\psi(r)=\psi_{a}(r)$ for $r \in(0,1)$ is a solution of (9)-(10) with $m=m(a)$. Reciprocally, to any bounded nontrivial radial solution of (9)(10) corresponds a solution of (22) with

$$
a=\psi(0)-\log \left(\frac{m}{\int_{\Omega} e^{-\psi} d x}\right) \in(0, \infty) .
$$

This proves that the set of bounded nontrivial radial solutions of (9)-(10) is parametrized by $a>0$. As a consequence, it is straightforward to check that the set of the solutions of $(9)-(10)$ in $(0, \infty) \times L^{\infty}(\Omega)$ is a connected branch parametrized by the map

$$
a \mapsto\left(m(a), \psi_{a}\right)
$$

when $\Omega$ is a ball.

\subsubsection{Annuli}

Our last example is the analysis of the radially symmetric problem (8)-(10) in annuli $\Omega=\left\{x \in \mathbb{R}^{d}: a<|x|<A\right\}, 0<a<A<\infty$. However, there are also nonradial solutions of (9)-(10) in certain cases, cf. [31] and the references therein. Notice here that there are no restrictions on the dimension. 


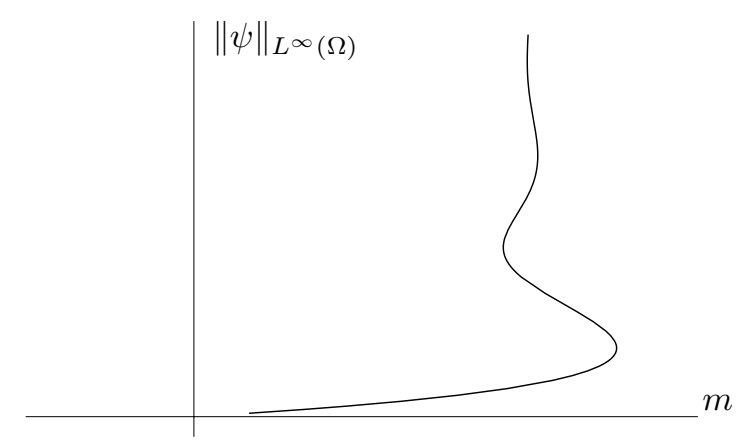

The bifurcation diagram in $(0, \infty) \times L^{\infty}(\Omega)$ when $\Omega$ is the unit disc in $\mathbb{R}^{3}$.

This case is qualitatively generic for $3 \leq d \leq 9$. Uniqueness is true only for $m>0$ small enough.

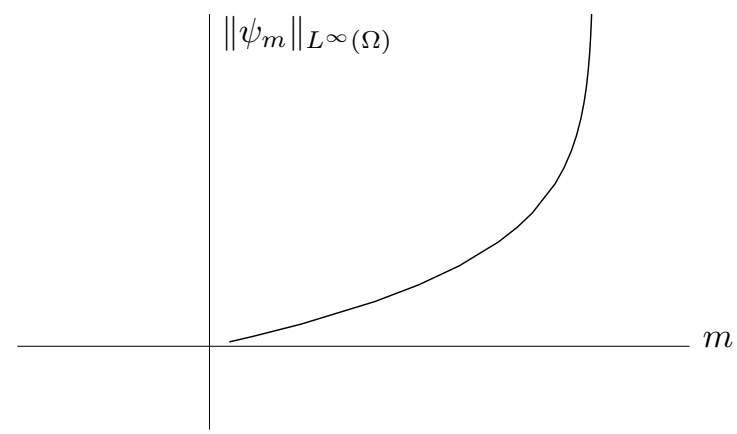

The bifurcation diagram in $(0, \infty) \times L^{\infty}(\Omega)$ when $\Omega$ is the unit disc in $\mathbb{R}^{d}$, with $d=10$.

Proposition 5.10 Assume that $d \geq 1$. In the annulus $\Omega=\left\{x \in \mathbb{R}^{d}: a<\right.$ $|x|<A\}$, given $E \in \mathbb{R}$ and $M>0$, radially symmetric solutions of (8)-(10) exist if $E / M^{2}>\ell_{1}$ for some $\ell_{1} \in \mathbb{R}$, and they do not exist if $E / M^{2}<\ell_{0} \leq \ell_{1}$. Moreover, if $d=1$ or 2 , then $\ell_{0}=\ell_{1}$.

Proof. The equation (9) for radially symmetric $\psi$, or (19) give $\left(\right.$ with $^{\prime}=\frac{d}{d r}$ )

$$
\begin{aligned}
\left(r^{d-1} \psi^{\prime}(r)\right)^{\prime}= & \lambda r^{d-1} e^{-\psi}, \quad a<r<A, \quad \lambda=m\left(\int_{\Omega} e^{-\psi} d x\right)^{-1}, \\
& \psi(a)=\psi(A)=0
\end{aligned}
$$

The function $r^{d-1} \psi^{\prime}(r)$ is increasing and because of the boundary conditions (24), $\psi^{\prime}(a) \leq 0 \leq \psi^{\prime}(A)$ holds by Hopf's lemma, so that $\psi^{\prime}(\alpha)=0$ for some $\alpha \in(a, A)$, and $\psi^{\prime}(r) \leq 0$ for $r \in[a, \alpha], \psi^{\prime}(r) \geq 0$ for $r \in[\alpha, A]$. Integrating 
(23) on either $[r, \alpha], a \leq r<\alpha$, or $[\alpha, r], \alpha<r \leq A$, we obtain

$$
\left|r^{d-1} \psi^{\prime}(r)\right|<\lambda \int_{a}^{A} \rho^{d-1} e^{-\psi(\rho)} d \rho=\frac{m}{\sigma_{d}} .
$$

Hence we have

$$
\left|\psi^{\prime}(r)\right|<\frac{m}{\sigma_{d}} r^{1-d}
$$

for all $a \leq r \leq A$, and

$$
\begin{aligned}
\int_{\Omega}|\nabla \psi|^{2} d x & =\sigma_{d} \int_{a}^{A} r^{d-1}\left|\psi^{\prime}(r)\right|^{2} d r \\
& <m^{2} \sigma_{d}^{-1} \int_{a}^{A} r^{1-d} d r \equiv C(a, A, d) m^{2}
\end{aligned}
$$

for all $m>0$, and this leads to the estimate

$$
m \geq \mathcal{F}(m)>m-C m^{2}
$$

with $C=\frac{1}{2} C(a, A, d)$. This proves the existence of $\ell_{0}$ and $\ell_{1}$.

We still have to prove that radial solutions belong to a single connected branch if $d=1$ or 2 .

If $d=1$, consider the auxiliary problem

$$
\begin{cases}\varphi^{\prime \prime}=e^{-\varphi}, & r>0 \\ \varphi(0)=0, & \varphi^{\prime}(0)=-\gamma\end{cases}
$$

for any given $\gamma>0$. Thus

$$
\frac{d}{d r}\left(\frac{1}{2}\left(\varphi^{\prime}(r)\right)^{2}+e^{-\varphi(r)}\right)=0
$$

so that it is easy to prove that there exists a $r(\gamma)=r>0$ such that $\varphi(r)=0$. The function $\psi=\psi^{\gamma}$ defined for $a<r<A$ by

$$
\psi^{\gamma}=\varphi\left(r(\gamma) \frac{r-a}{A-a}\right)
$$

is a solution of $(9)-(10)$ with

$$
m=m(\gamma) \equiv \frac{r(\gamma)}{A-a} \int_{0}^{r(\gamma)} e^{-\varphi(s)} d s=2 \frac{\gamma r(\gamma)}{A-a} .
$$


Reciprocally, to any radial bounded solution $\psi$ of $(9)$ corresponds a unique solution of (27) with $\gamma=-\left(\frac{1}{m} \int_{a}^{A} e^{-\psi(s)} d s\right)^{1 / 2} \psi^{\prime}(a)$.

If $d=2$, let us first transform (9)-(10) according to the change of variables given by

$$
\psi(r)=\chi(-\log r)-2 \log r,
$$

so that the function $\chi$ is a solution of

$$
\chi^{\prime \prime}=\frac{m}{2 \pi \int_{-\log A}^{-\log a} e^{-\chi(s)} d s} e^{-\chi}, \quad \chi(-\log a)=\chi(-\log A)=0,
$$

which is exactly the one-dimensional problem, and can therefore be parametrized by the solutions of (27).

The estimate (25) combined with the Leray-Schauder argument leads to a quick proof of existence of solutions of the problem (9)-(10) in annuli for each $m>0$.

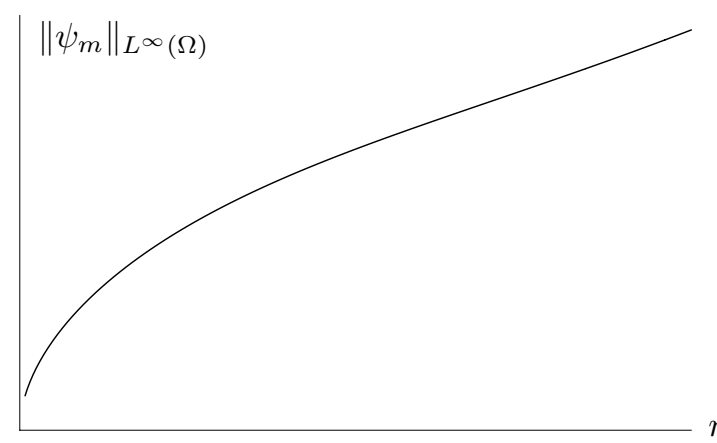

The bifurcation diagram in $(0, \infty) \times L^{\infty}(\Omega)$ when $\Omega$ is the annulus $\left\{x \in \mathbb{R}^{2}: 1<\right.$ $|x|<2\}$.

\subsection{Conclusion}

We believe that the situations described in examples for balls, strictly starshaped domains, and radially symmetric solutions in annuli are (qualitatively) generic for all bounded domains $\Omega \subset \mathbb{R}^{d}, d \geq 3$.

Conjecture. For every bounded domain in $\mathbb{R}^{d}, d \geq 3$, with piecewise smooth boundary, there exists $\ell_{0} \in \mathbb{R}$ such that the problem (8)-(10) has a solution 
with given $M>0$ and $E \in \mathbb{R}$ if $E / M^{2}>\ell_{0}$, and it does not have solutions if $E / M^{2}<\ell_{0}$.

Evidently, if $\Omega \subset \mathbb{R}^{d}$ is such that (9)-(10) has solutions for all $m>0$, i.e. $m_{\Omega}=\infty$, the analysis of the local behavior (17) of the graph of $\mathcal{E}\left(m, \psi_{m}\right)$ in a vicinity of $\langle 0,0\rangle$, and a global lower bound for $\mathcal{E}$, like (26) for some $C=C(\Omega)$, will imply the conjectured result, provided the set of the bounded solutions is a (single) connected branch.

It would be of interest to check for which domains $\inf _{m \in\left(0, m_{\Omega}\right)} \mathcal{F}(m)<0$, because in such a case $\ell_{0}<0$ is satisfied.

Acknowledgements. This research was partially supported by the KBN grant 2/P03A/011/19, the Austrian-Polish ÖAD-KBN Technical-Scientific Collaboration Agreement, the Austrian-French Scientific-Technical Collaboration Agreement AMADEE and the Wittgenstein Award 2000 of Peter Markowich financed by the Austrian Research Fund FWF.

\section{References}

[1] F. Bavaud, Equilibrium properties of the Vlasov functional: the generalized Poisson-Boltzmann-Emden equation, Rev. Mod. Phys. 63 no. 1 (1991), 129-148.

[2] P. Biler, J. Dolbeault, M. J. Esteban, G. Karch, Stationary solutions, intermediate asymptotics and large time behaviour of type II Streater's models, Adv. Diff. Eq. 6 (2001), 461-480.

[3] P. Biler, Existence and nonexistence of solutions for a model of gravitational interaction of particles, III, Colloq. Math. 68 (1995), 229-239.

[4] P. Biler, W. Hebisch, T. Nadzieja, The Debye system: existence and long time behavior of solutions, Nonlinear Analysis T. M. A. 23 (1994), 1189-1209.

[5] P. Biler, D. Hilhorst, T. Nadzieja, Existence and nonexistence of solutions for a model of gravitational interaction of particles, II, Colloq. Math. 67 (1994), 297-308. 
[6] P. Biler, A. Krzywicki, T. Nadzieja, Self-interaction of Brownian particles coupled with thermodynamic processes, Rep. Math. Phys. 42 (1998), 359-372.

[7] P. Biler, T. Nadzieja, Existence and nonexistence of solutions for a model of gravitational interaction of particles, I, Colloq. Math. 66 (1994), 319 334.

[8] P. Biler, T. Nadzieja, A nonlocal singular parabolic problem modelling gravitational interaction of particles, Adv. Diff. Eq. 3 (1998), 177-197.

[9] F. Bouchut, J. Dolbeault, On long time asymptotics of the VlasovFokker-Planck equation and of the Vlasov-Poisson-Fokker-Planck system with Coulombic and Newtonian potentials, Diff. Int. Eq. 8 (1995), $487-514$.

[10] S. Chandrasekhar, An Introduction to the Study of Stellar Structure, New York, Dover (1957).

[11] E. Caglioti, P. L. Lions, C. Marchioro, M. Pulvirenti, A special class of stationary flows for two-dimensional Euler equations: a statistical mechanics description, I \& II, Comm. Math. Phys. 143 (1992), 501-525 \& 174 (1995), 229-260.

[12] S.-Y. A. Chang, P. Yang, Conformal deformation of metrics on $S^{2}$, J. Diff. Geom. 27 (1988), 259-296.

[13] C. C. Chen, C. S. Lin, Blowup behavior of mean field type equation, Taiwanese J. Math. 4 (2000), 21-31.

[14] W. Ding, J. Jost, J. Li, G. Wang, Existence results for mean field equations, Ann. Inst. H. Poincaré, Analyse non linéaire 16 (1999), 653-666.

[15] J. Dolbeault, Free energy and solutions of the Vlasov-Poisson-FokkerPlanck system: external potential and confinement (large time behavior and steady states), J. Math. Pures Appl. (9) 78 (1999), 121-157.

[16] J. Dolbeault, F. Poupaud, A remark on the critical explosion parameter for a semilinear elliptic equation in a generic domain using an explosion time of an ordinary differential equation, Nonlinear Analysis T. M. A. 24 (1995), 1149-1162. 
[17] G. L. Eyink, H. Spohn, Negative-temperature states and large-scale, long-lived vortices in two-dimensional turbulence, J. Stat. Phys. 70 (1993), 833-886.

[18] Th. Gallouët, F. Mignot, J.-P. Puel, Quelques résultats sur le problème $-\Delta u=\lambda e^{u}\left[A\right.$ few results on the equation $\left.-\Delta u=\lambda e^{u}\right]$, C. R. Acad. Sci. Paris Sér. I Math. 307 (1988), 289-292.

[19] I. M. Gelfand, Some problems in the theory of quasi-linear equations, Amer. Math. Soc. Transl. 29 (1963), 295-381.

[20] B. Gidas, W.-M. Ni, L. Nirenberg, Symmetry and related properties via the maximum principle, Comm. Math. Phys. 68 (1979), 209-243.

[21] D. D. Joseph, T. S. Lundgren, Quasilinear problems driven by positive sources, Arch. Rat. Mech. Anal. 49 (1973), 241-269.

[22] A. Jüngel, Quasi-hydrodynamic Semiconductor Equations, PNLDE 41, Birkhäuser, Basel, Boston, 2000.

[23] M. K. H. Kiessling, Statistical mechanics of classical particles with logarithmic interactions, Comm. Pure Appl. Math. 46 (1993), 27-56.

[24] A. Krzywicki, T. Nadzieja, A note on the Poisson-Boltzmann equation, Zastos. Mat. 21 (1993), 591-595.

[25] A. Krzywicki, T. Nadzieja, Steady states for a model of interacting particles, Appl. Math. Lett. 13 (2000), 113-117.

[26] P. L. Lions, A. Majda, Equilibrium statistical theory for nearly parallel vortex filaments, Comm. Pure Appl. Math. 53 (2000), 76-142.

[27] J. Messer, H. Spohn, Statistical mechanics of the isothermal LaneEmden equation, J. Stat. Phys. 29 (1982), 561-578.

[28] F. Mignot, J.-P. Puel, Quelques résultats sur un problème elliptique avec non linéarité exponentielle [Some results on an elliptic problem with exponential nonlinearity], Équations aux dérivées partielles et applications, 683-704, Gauthier-Villars, Éd. Sci. Méd. Elsevier, Paris (1998). 
[29] T. Nadzieja, A note on nonlocal equations in mathematical physics, "Disordered and Complex Systems", London - King's College, July 2000, P. Sollich et al., ed., AIP Conference Proceedings 553, Melville, NY, 2001, 255-259.

[30] T. Nadzieja, A. Raczyński, Radially symmetric solutions of the PoissonBoltzmann equation with a given energy, Appl. Math. (Warsaw) 27 (2000), 465-473.

[31] K. Nagasaki, T. Suzuki, Radial solutions for $\Delta u+\lambda e^{u}=0$ on annuli in higher dimensions, J. Diff. Eq. 100 (1992), 137-161.

[32] L. Onsager, Statistical hydrodynamics, Suppl. Nuovo Cim. (9) 6 no. 2 (1949), 279-287.

[33] T. Padmanabhan, Statistical mechanics of gravitating systems, Physics Reports 188 no. 5 (1990), 285-362.

[34] S. I. Pohozaev, Eigenfunctions of the equation $\Delta u+\lambda f(u)=0$, Soviet Math. Dokl. 5 (1965), 1408.

[35] F. Rellich, Darstellung der Eigenwerte von $\Delta u+\lambda u=0$ durch ein Randintegral, Math. Z. 46 (1940), 635-636.

[36] C. Rosier, Problème de Cauchy pour une équation parabolique modélisant la relaxation des systèmes stellaires auto-gravitants, C. R. Acad. Sci Paris 332 (2001), 903-908.

[37] K. Schmitt, Positive solutions of semilinear elliptic boundary value problems, Topological methods in differential equations and inclusions (Montreal, PQ, 1994), 447-500, NATO Adv. Sci. Inst. Ser. C Math. Phys. Sci., 472, Kluwer Acad. Publ., Dordrecht, 1995.

[38] M. Smoluchowski, Drei Vorträge über Diffusion, Brownsche Molekularbewegung und Koagulation von Kolloidteilchen, Phys. Zeit. 17 (1916), $557-571,585-599$.

[39] R. F. Streater, A gas of Brownian particles in stochastic dynamics, J. Stat. Phys. 88 (1997), 447-469.

[40] R. F. Streater, Dynamics of Brownian particles in a potential, J. Math. Phys. 38 (1997), no. 9, 4570-4575. 
[41] R. F. Streater, Nonlinear heat equations, Rep. Math. Phys. 40 (1997), $557-564$.

[42] R. F. Streater, The Soret and Dufour effects in statistical dynamics, Proc. R. Soc. London A 456 (2000), 205-211.

[43] T. Suzuki, Global analysis for a two-dimensional elliptic eigenvalue problem with the exponential nonlinearity, Ann. Inst. H. Poincaré, Analyse non linéaire 9 (1992), 367-398.

[44] G. Wolansky, On steady distributions of self-attracting clusters under friction and fluctuations, Arch. Rat. Mech. Anal. 119 (1992), 355-391. 